The Astrophysical Journal, 652:1416-1422, 2006 December 1

(C) 2006. The American Astronomical Society. All rights reserved. Printed in U.S.A.

\title{
VERY EARLY OPTICAL AFTERGLOWS OF GAMMA-RAY BURSTS: EVIDENCE FOR RELATIVE PAUCITY OF DETECTION
}

\author{
Peter W. A. Roming, ${ }^{1}$ Patricia Schady, ${ }^{1,2}$ Derek B. Fox, ${ }^{1,3}$ Bing Zhang, ${ }^{4}$ Enwei Liang, ${ }^{4}$ Keith O. Mason, ${ }^{2}$ Evert Rol, ${ }^{5}$ \\ David N. Burrows, ${ }^{1}$ Alex J. Blustin, ${ }^{2}$ Patricia T. Boyd, ${ }^{6,7}$ Peter Brown, ${ }^{1}$ Stephen T. Holland, ${ }^{6,8}$ Katherine McGowan, ${ }^{2}$ \\ Wayne B. Landsman, ${ }^{6}$ Kim L. Page, ${ }^{5}$ James E. Rhoads, ${ }^{9}$ Simon R. Rosen, ${ }^{2}$ Daniel Vanden Berk, ${ }^{1}$ Scott D. Barthelmy, ${ }^{6}$ \\ Alice A. Breeveld, ${ }^{2}$ Antonino Cucchiara, ${ }^{1}$ Massimiliano De Pasquale, ${ }^{2}$ Edward E. Fenimore, ${ }^{10}$ Neil Gehrels, ${ }^{6}$ \\ Caryl Gronwall, ${ }^{1}$ Dirk Grupe, ${ }^{1}$ Michael R. Goad, ${ }^{5}$ Mariya Ivanushina,,${ }^{11}$ Cynthia James, ${ }^{2}$ Jamie A. Kennea, \\ Shiho Kobayashi, ${ }^{1}$ Vanessa Mangano, ${ }^{12}$ Peter Mészáros, ${ }^{1}$ Adam N. Morgan, ${ }^{1}$ John A. Nousek, ${ }^{1}$ \\ Julian P. Osborne, ${ }^{5}$ David M. Palmer, ${ }^{10}$ Tracey Poole, ${ }^{2}$ Martin D. Still, $, 8,8$ \\ Gianpiero Tagliaferri, ${ }^{13}$ and Silvia Zane ${ }^{2}$ \\ Received 2005 December 13; accepted 2006 August 10
}

\begin{abstract}
Very early observations with the Swift satellite of $\gamma$-ray burst (GRB) afterglows reveal that the optical component is not detected in a large number of cases. This is in contrast to the bright optical flashes previously discovered in some GRBs (e.g., GRB 990123 and GRB 021211). Comparisons of the X-ray afterglow flux to the optical afterglow flux and prompt $\gamma$-ray fluence is used to quantify the seemingly deficient optical, and in some cases X-ray, light at these early epochs. This comparison reveals that some of these bursts appear to have higher than normal $\gamma$-ray efficiencies. We discuss possible mechanisms and their feasibility for explaining the apparent lack of early optical emission. The mechanisms considered include, foreground extinction, circumburst absorption, Ly $\alpha$ blanketing and absorption due to high-redshift, low-density environments, rapid temporal decay, and intrinsic weakness of the reverse shock. Of these, foreground extinction, circumburst absorption, and high redshift provide the best explanations for most of the nondetections in our sample. There is tentative evidence of suppression of the strong reverse shock emission. This could be because of a Poynting flux-dominated flow or a pure nonrelativistic hydrodynamic reverse shock.
\end{abstract}

Subject heading: gamma rays: bursts

\section{INTRODUCTION}

The afterglow discoveries of 1997 revealed that gamma-ray bursts (GRBs) are the brightest explosions in the universe. They occur at cosmological distances (Metzger et al. 1997) and produce long-lived emission across the electromagnetic spectrum, from the X-ray band (e.g., Costa et al. 1997) through optical (e.g., Van Paradijs et al. 1997) to radio (e.g., Frail et al. 1997) wavelengths. While X-ray afterglows of GRBs are detected in nearly all cases (De Pasquale et al. 2003, hereafter D03; Burrows et al.

\footnotetext{
1 Department of Astronomy and Astrophysics, Pennsylvania State University, 525 Davey Lab, University Park, PA 16802; roming@astro.psu.edu.

2 Mullard Space Science Laboratory, University College London, Holmbury St. Mary, Dorking, Surrey RH5 6NT, UK.

3 Division of Physics, Mathematics and Astronomy, 105-24, California Institute of Technology, Pasadena, CA 91125.

4 Department of Physics, University of Nevada, Las Vegas, 4505 Maryland Parkway, Las Vegas, NV 89154-4002.

5 Department of Physics and Astronomy, University of Leicester, University Road, Leicester LE1 7RH, UK.

6 NASA/Goddard Space Flight Center, Greenbelt, MD 20771.

7 Joint Center for Astrophysics, University of Maryland, 1000 Hilltop Circle, Baltimore, MD 21250

${ }^{8}$ Universities Space Research Association, 10227 Wincopin Circle, Suite 212, Columbia, MD 21044

9 Space Telescope Science Institute, 3700 San Martin Drive, Baltimore, MD 21218.

${ }^{10}$ Los Alamos National Laboratory, P.O. Box 1663, Los Alamos, NM 87545

11 Department of Physics and Astronomy, Brigham Young University, N283 ESC, Provo, UT 84602.

12 INAF-Instituto di Astrofisica Spaziale e Fisica Cosmica Sezione di Palermo, via Ugo La Malfa 153, I-90146 Palermo, Italy.

13 INAF-Osservatorio Astronomico di Brera, Via Bianchi 46, 23807 Merate, Italy.
}

2006), their optical afterglows often remain undetected even in deep searches (Roming \& Mason 2006, hereafter RM06). Identifying and characterizing these "dark" bursts has been difficult due to the delays (typically hours) occurring between the detection of the GRB and the execution of the first ground-based observations.

Classical explanations for dark bursts include: absorption at the burst location (Piro et al. 2002; Lazzati et al. 2002; Djorgovski et al. 2001; Fynbo et al. 2001, hereafter F01), low-density environment (Frail et al. 1999; Groot et al. 1998a; Taylor et al. 2000), rapid temporal decay (Groot et al. 1998a), foreground extinction (Taylor et al. 1998), Ly $\alpha$ blanketing and absorption due to high redshift (F01; Groot et al. 1998b), or intrinsic faintness (Lazzati et al. 2002; F01). Previous authors have quantified the degree of optical darkness expected based on the upper limit on the afterglow flux (ULAF; Rol et al. 2005a) or the optical-to-X-ray spectral index ( $\beta_{\mathrm{OX}}$; Jakobsson et al. 2004, hereafter J04), and show that most "dark" bursts can be explained by adverse observing conditions.

Observations of GRBs with NASA's Swift satellite (Gehrels et al. 2004) are providing prompt few-arcminute $\gamma$-ray localizations, rapid few-arcsecond X-ray positions, and rapid and extensive follow-up in the X-ray, UV, optical, and radio bands (e.g., Gehrels et al. 2005; Cameron \& Frail 2005). Thirteen of these bursts include extraordinary optical upper limits at very early epochs after the burst. This is in contrast to the bright optical flashes discovered to accompany some GRBs (e.g., Akerlof et al. 1999; F01; Fox et al. 2003; Li et al. 2003) in the pre-Swift era.

In this paper we report the very early observations of GRB afterglows by the Swift UV/Optical Telescope (UVOT; Roming et al. 2005a) and the associated very tight early optical upper limits. 
TABLE 1

Basic Properties of the 19 Bursts in the Swift UVOT Sample

\begin{tabular}{|c|c|c|c|c|c|c|c|c|c|c|c|}
\hline $\begin{array}{l}\text { GRB } \\
(1)\end{array}$ & $\begin{array}{l}\log F_{\mathrm{X}, 1} \\
\text { (2) }\end{array}$ & $\begin{array}{c}\log S_{\gamma} \\
\text { (3) }\end{array}$ & $\begin{array}{c}\log f_{R, 1} \\
(\mu \mathrm{Jy}) \\
(4)\end{array}$ & $\begin{array}{l}t_{U} \\
(\mathrm{~s}) \\
(5)\end{array}$ & $\begin{array}{c}\Delta T_{U} \\
(\mathrm{hrs}) \\
(6)\end{array}$ & $\begin{array}{c}m_{U} \\
(\mathrm{mag}) \\
(7)\end{array}$ & $\begin{array}{c}A_{V} \\
\text { (mag) } \\
(8)\end{array}$ & $\begin{array}{c}\Delta T_{G} \\
\text { (days) } \\
(9)\end{array}$ & $\begin{array}{c}m_{G} \\
(\mathrm{mag}) \\
(10)\end{array}$ & $\begin{array}{c}z \\
(11)\end{array}$ & $\begin{array}{c}\text { References } \\
\text { (12) }\end{array}$ \\
\hline $050215 \mathrm{~B}^{\mathrm{a}} \ldots \ldots \ldots \ldots \ldots$ & 1.70 & -0.64 & 1.76 & 100 & 0.485 & 18.44 & 0.08 & 0.41 & $K=20.2$ & $\ldots$ & $1,2,3$ \\
\hline $050219 \mathrm{~A}^{\mathrm{a}} \ldots \ldots \ldots \ldots \ldots$ & 1.48 & 0.97 & 0.63 & 100 & 0.026 & 18.10 & 0.50 & $\ldots$ & $\ldots$ & $\ldots$ & 4,5 \\
\hline $050219 B^{\mathrm{a}} \ldots \ldots \ldots \ldots \ldots \ldots$ & 2.58 & 1.36 & 2.27 & 100 & 0.871 & 17.81 & 0.09 & 4.14 & $K_{s}=21.5$ & $\ldots$ & $6,7,8,9,10$ \\
\hline $050223^{\mathrm{a}} \ldots \ldots$ & 0.13 & -0.13 & 2.11 & 100 & 0.773 & 18.08 & 0.28 & $\ldots$ & $\ldots$ & $\ldots$ & 11,12 \\
\hline $050315^{\mathrm{a}} \ldots \ldots \ldots \ldots \ldots \ldots \ldots$ & 2.42 & 0.63 & 0.66 & 100 & 0.024 & 17.94 & 0.15 & 0.48 & $R=20.9$ & 1.95 & $13,14,15,16$ \\
\hline $050326^{\mathrm{a}} \ldots \ldots \ldots \ldots \ldots \ldots$ & 2.28 & 1.28 & 2.01 & 100 & 0.905 & 18.51 & 0.12 & $\ldots$ & $\ldots$ & $\ldots$ & $17,18,19$ \\
\hline $050410^{\mathrm{a}} \ldots$. & 1.53 & 0.84 & 2.10 & 100 & 0.528 & 17.70 & 0.34 & $\ldots$ & $\ldots$ & $\ldots$ & 20,21 \\
\hline $050412^{\mathrm{a}} \ldots \ldots \ldots \ldots \ldots \ldots$ & 1.34 & 0.32 & 0.57 & 100 & 0.026 & 18.25 & 0.06 & $\ldots$ & $\ldots$ & $\ldots$ & 22,23 \\
\hline $050421^{\mathrm{a}} \ldots \ldots \ldots \ldots \ldots \ldots$ & -0.71 & -0.74 & 1.57 & 100 & 0.031 & 15.95 & 2.51 & $\ldots$ & $\ldots$ & $\ldots$ & 24 \\
\hline $050422^{\mathrm{a}} \ldots \ldots \ldots \ldots \ldots \ldots$ & 0.33 & 0.07 & 2.50 & 100 & 0.031 & 13.62 & 4.31 & $\ldots$ & $\ldots$ & $\ldots$ & $25,26,27$ \\
\hline $050502 \mathrm{~B}^{\mathrm{a}} \ldots \ldots \ldots \ldots \ldots$ & 1.17 & -0.11 & 0.49 & 100 & 0.017 & 17.98 & 0.09 & 0.02 & $I=19.8$ & $\ldots$ & $28,29,30,31$ \\
\hline $050505^{\mathrm{a}} \ldots \ldots \ldots \ldots \ldots \ldots$ & 2.79 & 0.61 & 2.22 & 100 & 0.782 & 17.81 & 0.06 & 0.32 & $R=21.5$ & 4.27 & $32,33,34,35,36$ \\
\hline $050509 \mathrm{~A}^{\mathrm{a}} \ldots \ldots \ldots \ldots \ldots$ & 1.92 & -0.34 & 1.23 & 91 & 0.014 & 15.95 & 1.86 & $\ldots$ & $\ldots$ & $\ldots$ & 37,38 \\
\hline $050318 \ldots \ldots \ldots \ldots \ldots \ldots \ldots$ & 2.25 & 0.32 & 2.08 & 100 & 0.910 & 17.25 & 0.06 & 0.34 & $R=\#$ & 2.80 & $39,40,41,42$ \\
\hline $050319 \ldots \ldots \ldots \ldots \ldots \ldots \ldots$ & 2.58 & -0.10 & 1.81 & 100 & 0.024 & 17.09 & 0.03 & 0.33 & $B=20.9$ & 3.24 & $43,44,45,46,47,48$ \\
\hline $050406 \ldots \ldots \ldots \ldots \ldots \ldots$ & 0.54 & -1.02 & 0.96 & 100 & 0.024 & 19.41 & 0.06 & 0.32 & $r \sim 22$ & 2.44 & $49,50,51,52$ \\
\hline 050416А ................... & 2.52 & -0.42 & 1.44 & 100 & 0.022 & 18.90 & 0.09 & 0.64 & $R=20.9$ & 0.65 & $53,54,55,56$ \\
\hline $050525 \mathrm{~A} \ldots \ldots \ldots \ldots \ldots$ & 2.26 & 1.30 & 2.65 & 100 & 0.024 & 13.19 & 0.31 & 0.22 & $B=18.8$ & 0.69 & $57,58,59,60$ \\
\hline $050603 \ldots \ldots \ldots \ldots \ldots \ldots \ldots$ & 2.93 & 1.11 & 4.27 & 100 & 9.230 & 18.01 & 0.09 & 0.14 & $R=16.5$ & 2.82 & $61,62,63,64,65$ \\
\hline
\end{tabular}

Note.-Col. (1): GRB name; col. (2): X-ray flux in the $2-10 \mathrm{keV}$ range at $1 \mathrm{hr}$ after the BAT trigger (hereafter referred to as the trigger), in units of $10^{-13}$ ergs $\mathrm{cm}^{-2} \mathrm{~s}^{-1}$;

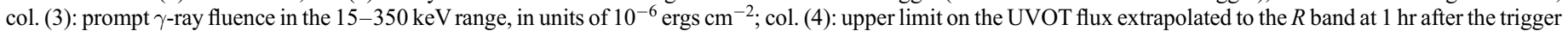

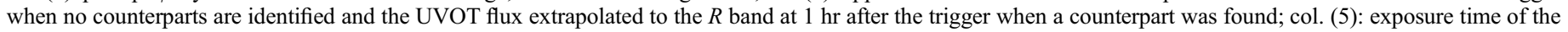

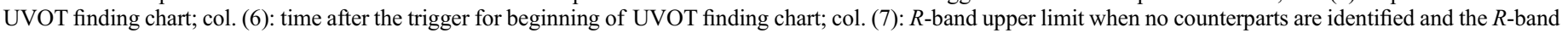

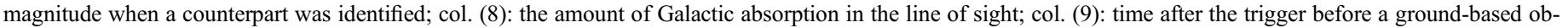

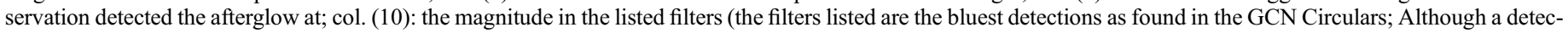
tion was claimed, no magnitude was provided in the GCN Circulars for GRB 050318); col. (11): redshift determined from ground-based or UVOT data; col. (12): references.

a These are GRBs with no UVOT counterparts identified.

References.- (1) Page et al. 2005; (2) Roming et al. 2005b; (3) Tanvir et al. 2005a; (4) Romano et al. 2005; (5) Schady et al. 2005b; (6) Burrows et al. 2005b; (7) Cummings et al. 2005a; (8) Ivanushkina et al. 2005; (9) D’Avanzo et al. 2005; (10) Berger et al. 2005b; (11) Giommi et al. 2005; (12) Gronwall et al. 2005; (13) Parsons et al. 2005a; (14) Rosen et al. 2005b; (15) Cobb \& Bailyn 2005a; (16) Kelson \& Berger 2005; (17) Markwardt et al. 2005; (18) Cummings et al. 2005b; (19) Holland et al. 2005; (20) La Parola et al. 2005; (21) Boyd et al. 2005a; (22) Cummings et al. 2005c; (23) Roming et al. 2005c; (24) Barbier et al. 2005b; (25) Barbier et al. 2005a; (26) Suzuki et al. 2005; (27) McGowan et al. 2005a; (28) Falcone et al. 2005; (29) Cummings et al. 2005d; (30) Cenko et al. 2005a; (31) Cenko et al. 2005b; (32) Hurkett et al. 2005a; (33) Hullinger et al. 2005; (34) Rosen et al. 2005a; (35) Tanvir et al. 2005b; (36) Berger et al. 2005a; (37) Hurkett et al. 2005b; (38) Barbier et al. 2005c; (39) Krimm et al. 2005a; (40) McGowan et al. 2005b; (41) Mulchaey \& Berger 2005; (42) Still et al. 2005; (43) Rykoff et al. 2005a; (44) Krimm et al. 2005b; (45) Boyd et al. 2005b; (46) Sharapov et al. 2005; (47) Fynbo et al. 2005; (48) Mason et al. 2006; (49) Parsons et al. 2005b; (50) Rol et al. 2005b; (51) Berger et al. 2005c; (52) Schady et al. 2006a; (53) Sakamoto et al. 2005; (54) Schady et al. 2005a; (55) Kahharov et al. 2005; (56) Cenko et al. 2005c; (57) Rykoff et al. 2005b; (58) Cummings et al. 2005e; (59) Cobb \& Bailyn 2005b; (60) Blustin et al. 2006; (61) Retter et al. 2005; (62) Fenimore et al. 2005; (63) Brown et al. 2005; (64) Berger \& McWilliam 2005; (65) Berger \& Becker 2005.

In $\S 2$ we present the observations and data reduction methods. In $\S 3$ we combine the Swift optical, X-ray, and $\gamma$-ray data sets to quantify the apparent lack of optical, and in few instances $\mathrm{X}$-ray, flux at these early epochs. We discuss possible mechanisms for explaining the apparent lack of early optical afterglows. In $\S 4$ we provide our conclusions.

\section{OBSERVATIONS AND REDUCTIONS}

Between 2005 January 24 and June 30, the Swift Burst Alert Telescope (BAT; Barthelmy et al. 2005) detected and localized 26 GRBs, which were thereafter observed by the Swift X-Ray Telescope (XRT; Burrows et al. 2005a) and UVOT. Identification of the fading X-ray afterglow in each of these cases has allowed the distribution of positions accurate to better than $6^{\prime \prime}$ within minutes to hours of the burst. The positions of 13 of these afterglows were observed with the UVOT less than $1 \mathrm{hr}$ after the burst, without optical counterparts being found (see Table 1 and Fig. 1). Seven of the 13 afterglows were observed by Swift within 2 minutes of the burst onset with no optical emission detected by UVOT. For comparison purposes, we have included six bursts that were detected by the UVOT (see Table 1).

Table 1 provides the basic properties of the 19 bursts in the Swift UVOT sample. The XRT data were processed using version 2.0 of the Swift software. Cleaned event lists were read into XSELECT, where source and background spectra and light curves were extracted. If the data appeared piled up in the photon-counting (PC) mode (i.e., if the count rate was $\gtrsim 0.6$ count $\mathrm{s}^{-1}$ ), an annular extraction region was used, with the inner radius dependent on the level of pile up; otherwise, a circle of radius $\sim 30$ pixels was chosen ( 1 pixel $=2$ ".36). The background files were obtained from nearby "source-free" areas of sky. The X-ray light curves were modeled with a combination of single and broken power laws and the models were then used to estimate the count rates at 1 and $11 \mathrm{hr}$. The spectra were fitted in XSPEC v11.3 and used to obtain a mean count rate to an unabsorbed flux conversion. The gamma-ray fluence was calculated in the $15-350 \mathrm{keV}$ energy band using the best-fit spectral parameters. For GRBs observed with other telescopes, a break energy at $250 \mathrm{keV}$ with the low and high photon energy indices set to -1 and -2.3 , respectively (Band et al. 1993) was assumed. The $3 \sigma$ upper limit for each burst was ascertained using a $3^{\prime \prime}$ and $6^{\prime \prime}$ diameter aperture (for the optical and UV filters, respectively) around the XRT position and then correcting for Galactic extinction. All upper limits and magnitudes were determined using the UVOT $V$-filter finding chart, which occurs after the spacecraft has settled on the target. $R$-band values were derived from the UVOT $V$ bandpass by 


\section{Upper Limits on Swift UVOT Bursts}

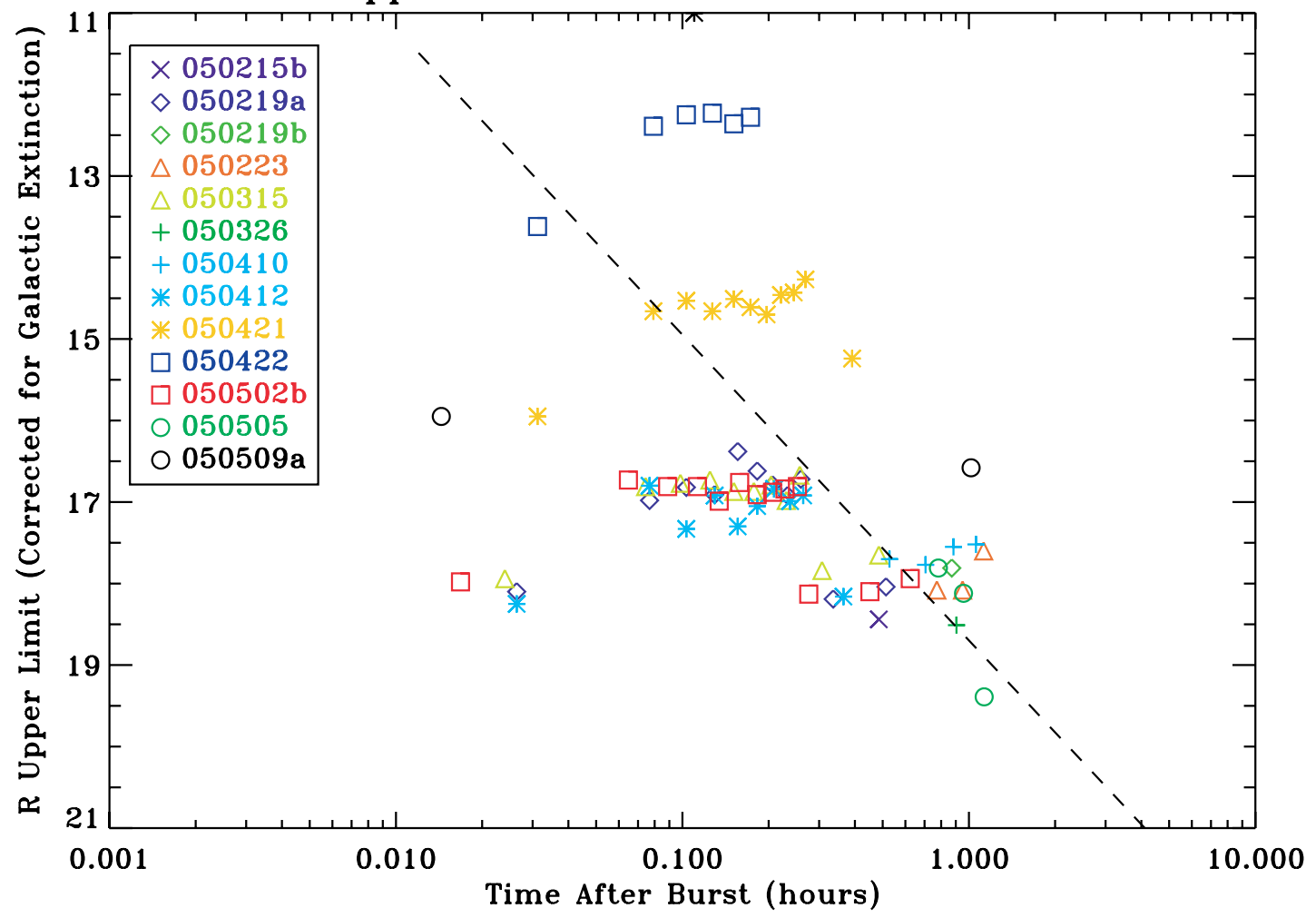

FIG. 1.-3 $\sigma$ UVOT-magnitude upper limit for each exposure corrected for galactic extinction and adjusted to the $R$ band. The UVOT prompt slew observation

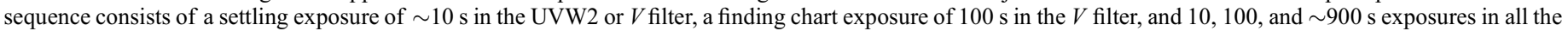

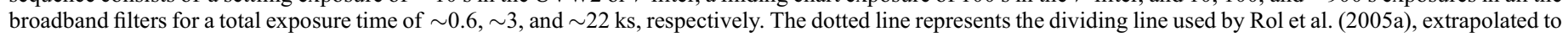
earlier times, which separates the dark bursts in their sample from practically all detected pre-Swift afterglows.

extrapolating to the effective $R$-band wavelength with the assumption of a $\nu^{-1}$ spectral shape. Errors on the $R$-band magnitudes range from \pm 0.22 to \pm 0.53 . The zero points from Fukugita et al. (1995) were used to convert the magnitude to fluxes. The bluest magnitude for each burst, established by ground-based observers, was obtained from the GRB Coordinate Network (GCN) Circulars (Barthelmy et al. 1995, 1998). Redshifts were obtained from both the GCN and UVOT observations.

\section{DISCUSSION}

BeppoSAX and HETE-2 satellite studies (J04) led us to expect that the UVOT would readily detect the prompt optical emission of most GRBs and their afterglows. ${ }^{14}$ However, the upper limits provided here show the UVOT bursts are not detected at or bluewards of the $V$ band at these early times. The speed of response and depth of UVOT observations are superior to the observations of all but a few previous GRBs. Moreover, accurate XRT positions make us confident that no bright optical afterglows were masked due to confusion with bright, nearby stars. The sensitivity and multiwavelength nature of our observations thus offers a unique opportunity to study the optical deficiency of these early afterglows.

As an initial test of the optical deficiency of our sample we employed the quantified ULAF and $\beta_{\text {OX }}$ methods. The $\beta_{\text {OX }}$ method is a rapid technique for ascertaining the potential darkness of Swift bursts. Those bursts with $\beta_{\mathrm{OX}}<0.50$ are classified as dark, while those bursts in the range of $0.50<\beta_{\mathrm{OX}}<0.55$ are clas-

14 We point out that Kehoe et al. (2001) showed that not all GRBs have GRB 990123-like optical flashes. sified as potentially dark. The ULAF method is a more thorough approach to dark burst classification. It utilizes the temporal $(\alpha)$ and spectral $(\beta)$ indices for determining the minimum and maximum value for the electron index $(p)$. Eight cases of the standard afterglow model are tested, and the resulting maximum and minimum X-ray flux are extrapolated to the optical epoch. Optical flux that falls below the minimum extrapolated X-ray flux is considered dark. Using the ULAF method, we found that only GRB 050219B could be classified as dark (a determination of the darkness of GRB 050421 could not be made with the ULAF method). The $\beta_{\text {OX }}$ method revealed that GRBs 050315, 050319, 050326, 050412, and 050505 were classified as dark (RM06; see Fig. 2). Even though the ULAF or $\beta_{\mathrm{OX}}$ classification schemes do not categorize most bursts in our sample as dark, it does not explain why the early optical afterglows are apparently lacking. For both the $\beta_{\mathrm{OX}}$ and ULAF methods, it is assumed that GRBs follow the standard fireball model (RM06). Recent Swift observations show much more complicated features in the early afterglow phase (Nousek et al. 2006, hereafter N06; Zhang et al. 2006a, hereafter Z06a), which calls for a more detailed study of the optical deficiency in the early phases.

To quantify the apparent lack of optical and X-ray light at these early epochs in the Swift bursts, we combine broadband UVOT observations with BAT and XRT data, and compare the burst's $\mathrm{X}$-ray afterglow flux at $1 \mathrm{hr}$ after the burst $\left(F_{\mathrm{X}, 1}\right)$ to its prompt $\gamma$-ray fluence $\left(S_{\gamma}\right)$ and the optical afterglow flux at $1 \mathrm{hr}\left(f_{R, 1}\right)$. This is done because the ratio $S_{\gamma} / S_{\mathrm{X}, 1}$ (where $S_{\mathrm{X}, 1}=F_{\mathrm{X}, 1}[1 \mathrm{hr}]$ is the $1 \mathrm{hr}$ $\mathrm{X}$-ray fluence) measures the $\gamma$-ray efficiency of the event, while the ratio $F_{\mathrm{X}, 1} / f_{R, 1}$ measures the amount of optical flux constrained by selective extinction (J04; D03); here $S_{\gamma}$ is the natural measure 


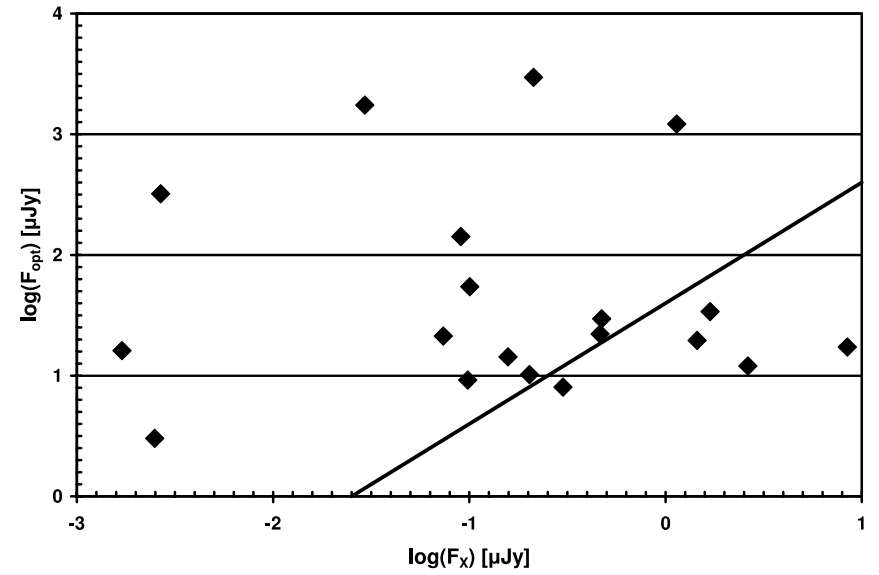

FIG. 2.-Optical-to-X-ray spectral index $\left(\beta_{\mathrm{OX}}\right)$ for the 19 bursts in the Swift UVOT sample. The dividing line represents $\beta_{\mathrm{OX}}=0.50$, which separates the dark bursts from the nondark bursts, as defined by Jakobsson et al. (2004). The bursts below the dividing line are GRBs 050315, 050319, 050326, 050412, and 050505 .

of the radiated energy of the GRB. The X-ray band flux at a certain epoch, e.g., $F_{\mathrm{X}, 1}$ (or fluence $S_{\mathrm{X}, 1}$ ) is a good indication of the fireball's blast-wave kinetic energy, especially if the cooling frequency is below the band (Kumar 2000; Freedman \& Waxman 2001; Berger et al. 2003; Lloyd-Ronning \& Zhang 2004). Although the fireball bulk Lorentz factor is different during the prompt $\gamma$-ray phase and $1 \mathrm{hr}$ after the burst trigger, the energy per solid angle along the viewing direction is essentially independent of the value of the bulk Lorentz factor and the jet structure (Zhang \& Mészáros 2002). The physical jet angle also does not change between the epoch of the prompt phase (when $S_{\gamma}$ is measured) and $1 \mathrm{hr}$ after the trigger (when $F_{\mathrm{X}, 1}$ and $S_{\mathrm{X}, 1}$ is measured). Finally, the value $S_{\gamma} / S_{\mathrm{X}, 1}$ only weakly depends on the unknown redshift $z$ when the X-ray band is above the cooling frequency (up to a correction factor of order unity; see, e.g., Zhang et al. 2006b, hereafter Z06b; Granot et al. 2006). As a result the ratio $S_{\gamma} / S_{\mathrm{X}, 1}$ (or $S_{\gamma} / F_{\mathrm{X}, 1}$ ) is an indication of the $\gamma$-ray efficiency.

We choose $1 \mathrm{hr}$ after the trigger as the fiducial epoch based on the following considerations. First, we want to compare the Swift data with the measurements of some pre-Swift bursts. The measurements of pre-Swift bursts usually happened several hours after the burst trigger. One therefore needs to extrapolate the late-time flux to early times (e.g., $1 \mathrm{hr}$ ). It is now clear that the early X-ray afterglow light curves typically show a shallow decay phase extending to about $1 \mathrm{hr}$ (N06; Z06a). Extrapolating the preSwift data to an epoch much earlier than $1 \mathrm{hr}$ inevitably leads to unreliable results, which would be inappropriate for directly comparing to the Swift data (cf. Figs. 3 and 4). On the other hand, the strength of Swift data are the early observations. In order to study the early afterglow properties it is unwise to extrapolate the data to later epochs. This is especially relevant for upper limits, since no decaying slope information is available. On the other hand, utilizing the Swift data at an epoch much earlier than $1 \mathrm{hr}$ induces large uncertainties, since the X-ray flux is dominated by flares and/or a rapid decline phase, which are thought to be associated with the central engine rather than the afterglow. We therefore find a compromise in taking $1 \mathrm{hr}$ as the fiducial epoch. One caveat is that this epoch is typically the transition time between the reverse shock component and the forward shock component for some optical flashes. However, these optical flashes are not commonly detected, especially in our sample. A detailed GRB efficiency study at a much earlier and a much later epoch has been presented elsewhere (Z06b).

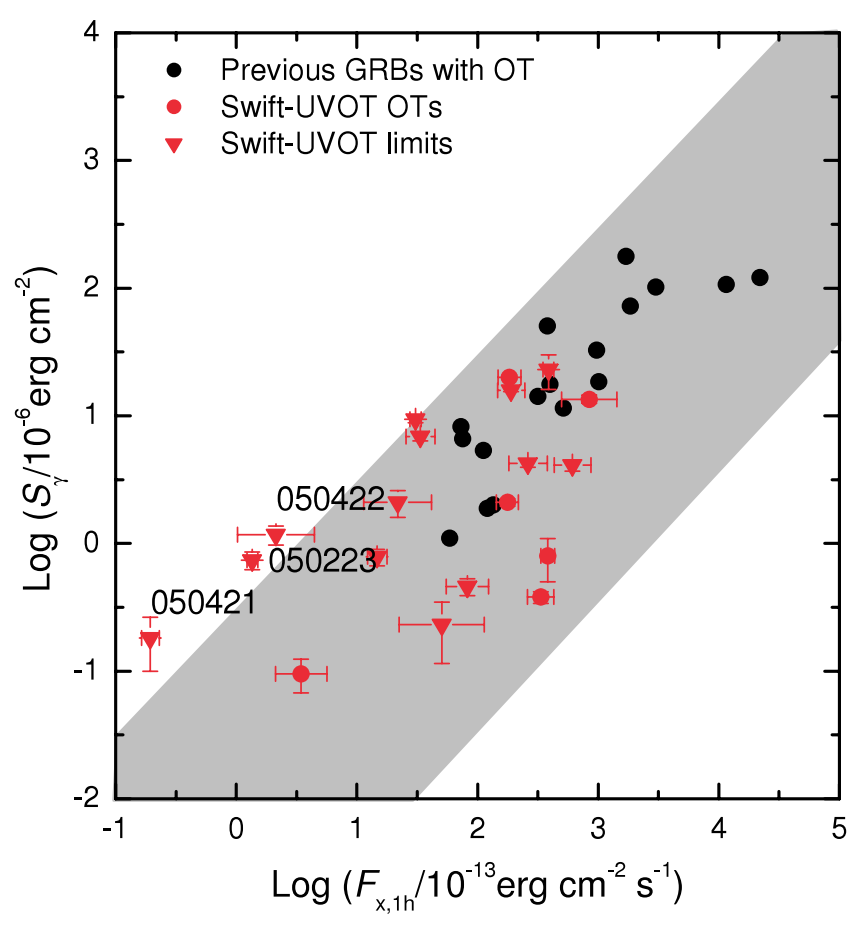

FIG. 3.-Distribution of Swift GRBs in the $F_{\mathrm{X}, 1}-S_{\gamma}$ plane. $F_{\mathrm{X}, 1}$ is the X-ray afterglow flux in the $2-10 \mathrm{keV}$ band at $1 \mathrm{hr}$ after the burst. $S_{\gamma}$ is the $\gamma$-ray fluence in the 15-350 keV energy band using the best-fit spectral parameters for Swift GRBs, or assuming a break energy at $250 \mathrm{keV}$ with the low and high photon energy indices set to -1 and -2.3 , respectively, for GRBs observed with other telescopes. Red and black circles represent the Swift GRBs and GRBs observed with other telescopes, respectively. Filled triangles denote those Swift GRBs undetected by UVOT. For non-Swift bursts, the X-ray data are taken from the literature (Berger et al. 2003). The X-ray value for GRB 050421 was calculated by extrapolating from the $100-500 \mathrm{~s}$ interval to $1 \mathrm{hr}$. The extrapolated value is consistent with observational upper limits on either side of $1 \mathrm{hr}$. The shaded region for the panel is twice the standard deviation $(2 \sigma)$ of $S_{\gamma} / F_{\mathrm{X}, 1}$ for optically-detected GRBs.

In Figure 3, we can see that most optically-detected GRBs prior to Swift fall into the gray band, which is twice the standard deviation $(2 \sigma)$ of $S_{\gamma} / F_{\mathrm{X}, 1}$ for optically-detected GRBs, thus seeming to support the idea that the efficiency of $\gamma$-ray production is roughly constant. In a similar fashion, the broadband nature of synchrotron emission implies that the ratio of X-ray and optical emission at a fixed epoch is roughly constant, except when selective extinction suppresses the optical flux (J04; D03). Indeed, the X-ray and optical fluxes of pre-Swift optically detected GRBs are correlated (Fig. 4).

Having quantified the apparent lack of optical and X-ray light, we now examine possible mechanisms. The first mechanism we consider is that of extinction by dust. For the case of foreground extinction, only Galactic absorption in the optical can be considered, since modeling intervening dust along the line of sight is problematic without an optical afterglow detection. ${ }^{15}$ The galactic absorption along the line of sight for most bursts in our sample is relatively small. However, in the case of GRBs 050421 , 050422, and 050509A the extinction is fairly large (see Table 1). In these cases it is not unreasonable to assume, at least in part, that the afterglows of these bursts are extincted due to intervening dust. For the case of circumburst absorption, preliminary results by Schady et al. (2006a) on a small sample of optically detected Swift bursts suggests that this effect is likely small for many Swift

15 A search for sources along the line of sight in the USNO-B catalog revealed that there were no sources coincident with the position of the optically deficient GRBs in our sample. 

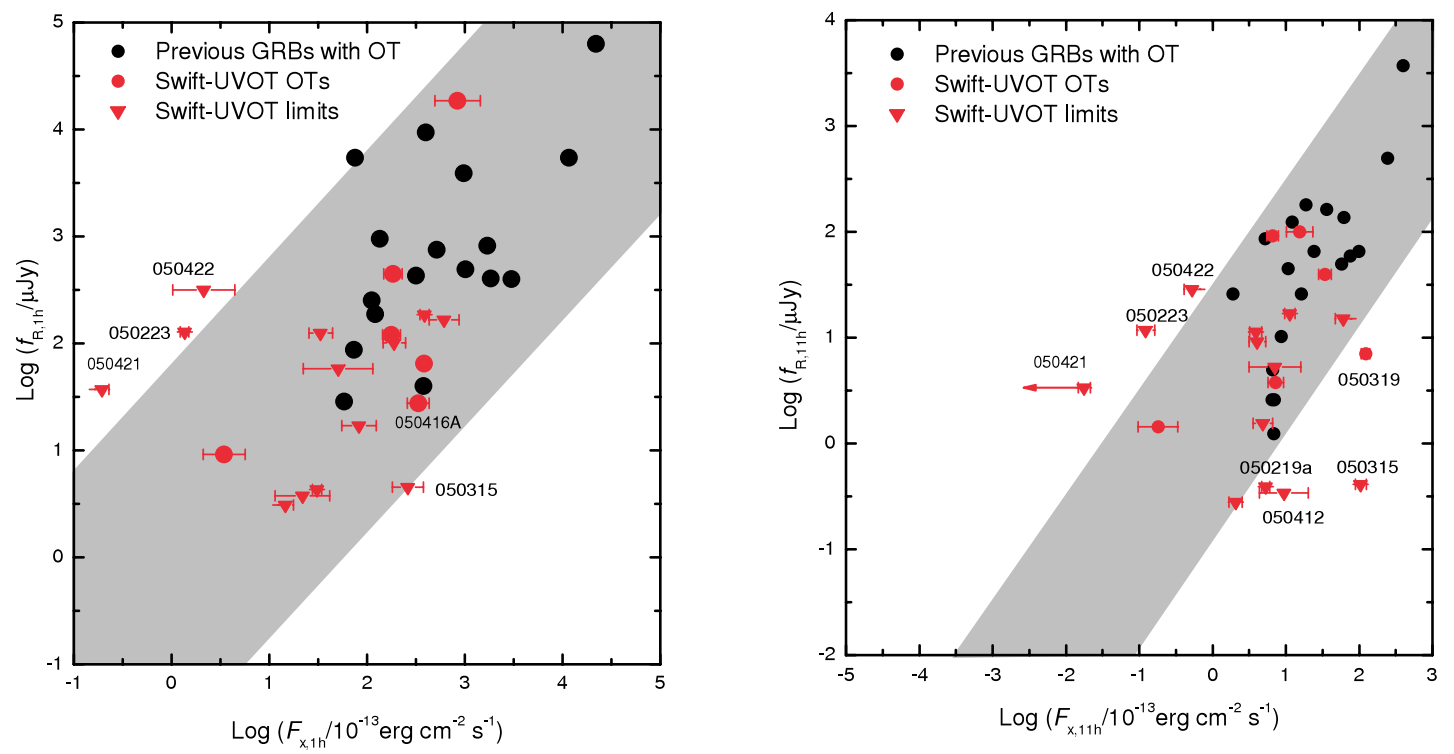

Fig. 4.-Distribution of Swift GRBs in the $F_{\mathrm{X}}-f_{R}$ plane at $1 \mathrm{hr}$ (left panel) and $11 \mathrm{hrs}$ (right panel) after the burst trigger, where $f_{R}$ is the R-band flux. Symbols are similar to those in Fig. 3. The $1 \mathrm{hr}$ optical upper limits for bursts without UVOT observations are derived by assuming a temporal decay index of -1 and the upper limits obtained by the ground-based telescopes. For three Swift bursts, GRBs 041223, 050117, and 050126, the upper limits are taken from Covino et al. (2005), Karimov et al. (2005), and Lipunov et al. (2005), respectively. For the pre-Swift bursts, these limits are taken from J04. The X-ray value at $11 \mathrm{hr}$ for GRB 050421 was calculated by extrapolating from $1 \mathrm{hr}$ using a slope of -1 . The shaded region for the panels is twice the standard deviation $(2 \sigma)$ of $F_{\mathrm{X}} / f_{R}$ for optically-detected GRBs.

bursts. This is in agreement with work done by Jakobsson et al. (2006), who suggest that only $20 \%$ of Swift bursts are highly obscured. This value implies that $2-3$ of the optically undetected bursts in our sample should be significantly obscured. An examination of Figure 4 (right panel), which investigates the relationship between $F_{\mathrm{X}}$ and $f_{R}$ at $11 \mathrm{hr}$ (which is a reasonable time for assuming that the cooling frequency has passed through the optical band), reveals that GRBs 050219A, 050315, and 050412 are probably obscured by circumburst dust. We note that the optically detected burst GRB 050319 is also below the $2 \sigma$ band.

The second mechanism we consider is that of Ly $\alpha$ blanketing and absorption due to high redshift. The UVOT is capable of detecting bursts out to $z \sim 5$. As an example, GRB 060522 was recently detected by UVOT in the white-light filter at $z=5.11$ (Holland 2006). The mean redshift of Swift localized bursts is $z_{\text {mean }}=2.8$ with at least $7 \%$ of the bursts located at $z>5$ (Jakobsson et al. 2006). Based on these criteria, and if we assume a fairly even distribution of bursts out to a redshift of $z \sim 5$ (see RM06), UVOT should detect most bursts in the first observations made with the white-light and $V$ filters out to $z \sim 4$. After this point the ability to detect an afterglow becomes increasingly more difficult (see Fig. 5). This equates to $\sim 30 \%$, or $3-4$ bursts in the sample, being optically undetected to UVOT due to Ly $\alpha$ blanketing and absorption at high-redshift.

An additional mechanism for suppressing the optical afterglow is a rapid temporal decay. The temporal decay profiles of a relatively small sample of detected Swift early optical afterglows are shallow $(\alpha \sim 1)$ in comparison to the canonical steep X-ray decay profiles $(\alpha \sim 3$; Tagliaferri et al. 2005; N06; O'Brien et al. 2006; Z06a; RM06; Yost et al. 2006). These shallow decays indicate that if detected bursts and optically deficient bursts behave similarly, then rapid temporal decay is not a significant consideration for our sample.

Another possibility to account for a low afterglow flux level is a low-density medium, if the X-ray band is below the cooling frequency. In fact about 1/4 of Swift X-ray afterglows are in this regime (Z06b). However, a low-density medium does not give a straightforward interpretation of the suppression of an optical flash, if the latter is attributed to a reverse-shock component. In fact, three bursts with an identified prompt optical flash, i.e., GRBs 990123 (Akerlof et al. 1999), 021211 (Fox et al. 2003; Li et al. 2003), and 041219A (Vestrand et al. 2005; Blake et al. 2005),

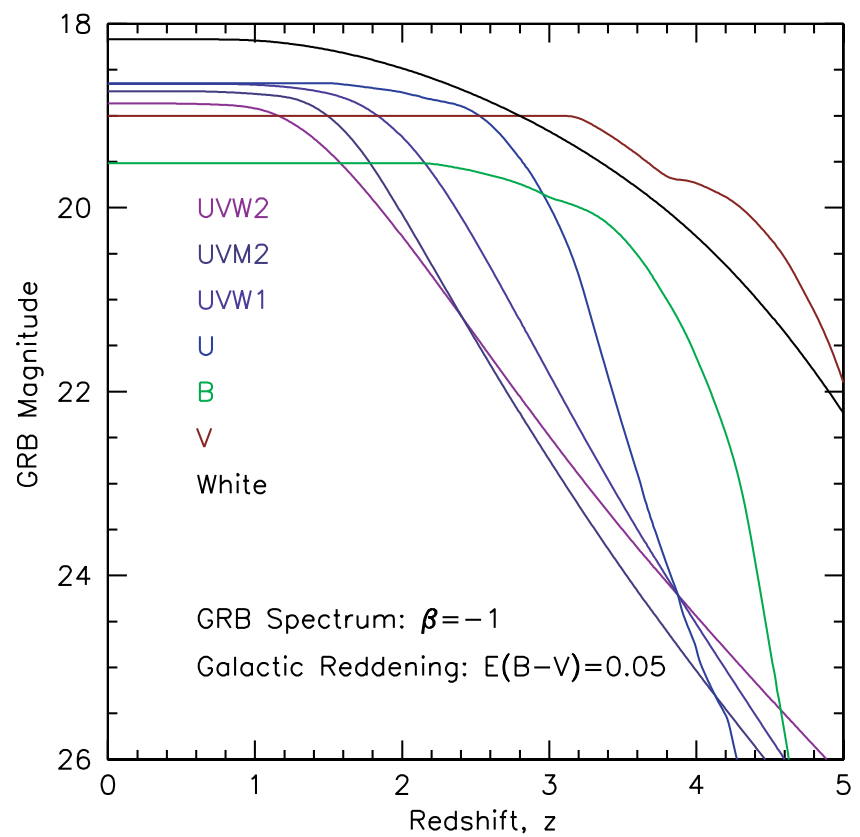

FIG. 5.-UVOT magnitude of a GRB vs. redshift for a "typical GRB" spectrum. A power-law SED, with spectral index $\beta=-1$ and Galactic $E(B-V)=$ 0.05 , which is normalized to have $V=19.0$ at $z=0$ was assumed. The current UVOT filter effective area curves, zero points, and counts-to-flux density calibrations were used derive the magnitudes. In the absence of Lyman absorption, the magnitudes would remain as they are at $z=0$. The spectrum is then run through Lyman extinction as a function of redshift, according to Madau (1995). The initial $(z=0)$ colors are due to $\beta$ and $E(B-V)$, but the falling magnitudes are due to Lyman absorption (Ly $\alpha, \operatorname{Ly} \beta, \operatorname{Ly} \gamma, \operatorname{Ly} \delta$, Lyman continuum). 
are all modeled to have a low-density environment (Panaitescu \& Kumar 2002; Kumar \& Panaitescu 2003; Fan et al. 2005).

It could be that the prompt optical radiation of reverse shocks is suppressed relative to that in the simple hydrodynamic models if the ejecta is Poynting dominated (e.g., Usov 1992; Thompson 1994; Mészáros \& Rees 1997a, 1997b; Spruit et al. 2001; Lyutikov \& Blandford 2003), since in that case the sound speed, or Alfvén speed in the ejecta, is close to the speed of light and the reverse compression wave fails to steepen into a shock. If in some bursts the outflows were indeed Poynting dominated, a significant suppression of the reverse shock emission would be expected (Zhang \& Kobayashi 2005). Conventional analyses of reverse shocks have in fact indicated that the ejecta may be more magnetized than the forward shock region (Zhang et al. 2003; Fan et al. 2002; Kumar \& Panaitescu 2003), but in general, there is no strong observational support for the presence of Poynting-dominated flows. However, more detailed investigations of purely hydrodynamic reverse shocks (i.e., not Poynting dominated) indicate that the strength of the optical emission from reverse shocks may be weaker than previously calculated (e.g., Kobayashi 2000; Nakar \& Piran 2004; Beloborodov 2005; Kobayashi et al. 2005; Uhm \& Beloborodov 2006; MacMahon et al. 2006). Thus, there are plausible physical reasons for which in the seven GRBs with tight very early optical limits, the reverse shock may be considerably suppressed.

Figure 3 highlights three Swift bursts, GRBs 050223, 050421, and 050422 , which are undetected optically and do not fall within the gray band. These bursts are deficient in X-ray emission compared to their $\gamma$-ray fluxes, as well as being optically faint. GRBs 050421 and 050422 , in particular, were observed very early $(<95 \mathrm{~s})$ after the burst, and were not detected by the UVOT to limits roughly 4-5 mag fainter than was previously possible (the observational evidence points strongly toward Galactic dust absorption suppressing the optical afterglow in these two bursts).

These GRBs appear to have a higher than normal $\gamma$-ray efficiency. In the case of GRB 050223 the $\gamma$-ray efficiency is estimated to be greater than $90 \%$ following the method derived by Lloyd-Ronning \& Zhang (2004).

One concern, related to the $\gamma$-ray efficiency of bursts, is the uncertainty in the constant nature of the kinetic energy of some bursts. Recent work suggests that the X-ray shallow decay phase is caused by energy injection (Rees \& Meszaros 1998; Z06a; N06; Panaitescu et al. 2006a, 2006b). Although this phase is typically over by $1 \mathrm{hr}$, there are cases in which the shallow decay phase continues for longer (i.e., GRB 050401; N06). By taking the afterglow kinetic energy after the injection is over, one gets a moderate $\gamma$-ray efficiency for most of the bursts (Z06b). As a result, if in some bursts the energy injection phase lasts much longer than $1 \mathrm{hr}$, taking the kinetic energy derived from $1 \mathrm{hr}$ would considerably underestimate the total kinetic energy of the burst. This would potentially inflate the $\gamma$-ray efficiency (Z06b). This statement is only valid if one believes that the total afterglow kinetic energy after the injection is over should be used to define the $\gamma$-ray efficiency. On the other hand, it could be that the kinetic energy right after the burst is the relevant quantity to define the efficiency.
If this is the case, most of the $\gamma$-ray efficiencies derived at $1 \mathrm{hr}$ are underestimated. Nonetheless, those with an extended injection phase would have measured efficiencies higher than the others, which can account for the apparent high-efficiency bursts in our sample. For a more detailed discussion of GRB efficiency, we refer the readers to $\mathrm{Z} 06 \mathrm{~b}$.

\section{CONCLUSION}

We have compared the X-ray afterglow flux to the optical afterglow flux and prompt $\gamma$-ray fluence of a sample of Swift GRBs to quantify the lack of optical afterglow emission. Using this quantification method, a few of these bursts manifest an apparent higher than normal $\gamma$-ray efficiency. Although most bursts proceed through the normal decay phase after $1 \mathrm{hr}$, some bursts manifest energy injection after this time, thus varying the kinetic energy of the fireball and potentially inflating the $\gamma$-ray efficiency. Further work is required in order to determine the true $\gamma$-ray efficiency of these bursts. Among various interpretations for the tight early UVOT upper limits (e.g., extinction, high redshift, etc.), most would not lead to the observed high $\gamma$-ray efficiency, since they only suppress the optical emission but not the X-ray emission.

An investigation into the possible mechanisms for the lack of optical emission points to $\sim 25 \%$ of the bursts in our sample being extincted by Galactic dust, while $\sim 25 \%$ are obscured by circumburst absorption (which is consistent with the $\sim 20 \%$ proposed by Jakobsson et al. 2006). An additional $\sim 30 \%$ are most likely attributable to Ly $\alpha$ blanketing and absorption at high redshift, although other mechanisms cannot be conclusively ruled out at this time. Rapid temporal decay as a valid mechanism is ruled out assuming that the decay profile of these optically deficient bursts behave similarly to their optical counterparts. A low-density environment is also eliminated as a possibility based on comparisons with optically detected afterglows generated in a low-density environment. While Poynting flux-dominated outflows could suppress the early optical afterglow, there are also indications that in normal hydrodynamic outflows the physics of the reverse shocks can, in many cases, lead to weaker optical emission than previously thought. More data and more detailed modeling is needed to test such explanations for the paucity of detected optical flashes.

We appreciate the comments from an anonymous referee that greatly improved the conclusions of this paper. We gratefully acknowledge the contributions from members of the Swift team at Pennsylvania State University, Mullar Space Science Laboratory (MSSL), University of Leicester, NASA/GSFC, INAF-OABr, and our subcontractors, who helped make this Observatory possible. We are also grateful to the Flight Operations Team for their support above and beyond the call of duty. This work is supported at Penn State by NASA contract NAS5-00136, at MSSL and Leicester by funding from the Particle Physics and Astronomy Research Council (PPARC), and at INAF-OABr by ASI contract I/R/039/04. The Los Alamos National Laboratory is operated by the University of California for the US Department of Energy (DOE).

\section{REFERENCES}

Akerlof, C., et al. 1999, Nature, 398, 400

Band, D., et al. 1993, ApJ, 413, 281

Barbier, L., Palmer, D., Burrows, D., Blustin, A., Krimm, H., Gehrels, N., McGowan, K., \& Chester, M. 2005a, GCN Circ. 3314

Barbier, L., Palmer, D., Burrows, D., Blustin, A., Markwardt, C., Gehrels, N., \& Roming, P. 2005b, GCN Circ. 3296

Barbier, L., et al. 2005c, GCN Circ. 3407
Barthelmy, S. D., Butterworth, P. S., Cline, T. L., \& Gehrels, N. 1998, in AIP Conf. Proc. 428, 4th Huntsville Symp. on Gamma-Ray Bursts, ed. C. A. Meegan, R. D. Preece, \& T. M. Koshut (New York: AIP), 139

Barthelmy, S. D., Butterworth, P., Cline, T. L., Gehrels, N., Fishman, G. J., Kouveliotou, C., \& Meegan, C. A. 1995, Ap\&SS, 231, 235

Barthelmy, S. D., et al. 2005, Space Sci. Rev., 120, 143

Beloborodov, A. M. 2005, ApJ, 618, L13 
Berger, E., \& Becker, G. 2005, GCN Circ. 3520

Berger, E., Cenko, S. B., Steidel, C., Reddy, N., \& Fox, D. B. 2005a, GCN Circ. 3368

Berger, E., Kelson, D., \& Gonzalez, S. 2005b, GCN Circ. 3047

Berger, E., Kulkarni, S. R., \& Frail, D. A. 2003, ApJ, 590, 379

Berger, E., \& McWilliam, A. 2005, GCN Circ. 3511

Berger, E., Oemler, G., \& Gladders, M. 2005c, GCN Circ., 3185

Blake, C. H., et al. 2005, Nature, 435, 181

Blustin, A. J., et al. 2006, ApJ, 637, 901

Boyd, P., et al. 2005a, GCN Circ. 3230 2005b, GCN Circ. 3129

Brown, P., et al. 2005, GCN Circ. 3516

Burrows, D. N., et al. 2005a, Space Sci. Rev., 120, 165 2005b, GCN Circ. 3042

2006, in AIP Conf. Proc. 836, 16th Maryland Astrophys. Conf. on Gamma-Ray Bursts in the Swift Era, ed. S. S. Holt, N. Gehrels, \& J. A. Nousek (Melville: AIP), 215

Cameron, P. B., \& Frail, D. A. 2005, GCN Circ. 3495

Cenko, S. B., Fox, D. B., Rich, J., Schmidt, B., Christiansen, J., \& Berger, E. 2005a, GCN Circ. 3357 2005b, GCN Circ., 3358

Cenko, S. B., Kulkarni, S. R., Gal-Yam, A., \& Berger, E. 2005c, GCN Circ. 3542

Cobb, B. E., \& Bailyn, C. D. 2005a, GCN Circ. 3104 2005b, GCN Circ. 3506

Costa, E., et al. 1997, Nature, 387, 783

Covino, S., et al. 2005, GCN Circ. 2907

Cummings, J., et al. 2005a, GCN Circ. 3044

2005b, GCN Circ. 3145

2005c, GCN Circ. 3237

2005d, GCN Circ. 3339

2005e, GCN Circ. 3479

D'Avanzo, P., et al. 2005, GCN Circ. 3064

De Pasquale, M., et al. 2003, ApJ, 592, 1018 (D03)

Djorgovski, S. G., et al. 2001, ApJ, 562, 654

Falcone, A., et al. 2005, GCN Circ. 3330

Fan, Y. Z., Dai, Z. G., Huang, Y. F., \& Lu, T. 2002, Chinese J. Astron. Astrophys., 2, 449

Fan, Y. Z., Zhang, B., \& Wei, D. M. 2005, ApJ, 628, L25

Fenimore, E., et al. 2005, GCN Circ. 3512

Fox, D. W., et al. 2003, ApJ, 586, L5

Frail, D., et al. 1997, Nature, 389, 261 1999, ApJ, 525, L81

Freedman, D. L., \& Waxman, E. 2001, ApJ, 547, 922

Fukugita, M., Shimasaku, K., \& Ichikawa, T. 1995, PASP, 107, 945

Fynbo, J. P. U., Hjorth, J., Jensen, B. L., Jakobsson, P., Moller, P., \& Näränen, J. 2005, GCN Circ. 3136

Fynbo, J. U., et al. 2001, A\&A, 369, 373 (F01)

Gehrels, N., et al. 2004, ApJ, 611, 1005 2005, Nature, 437, 851

Giommi, P., et al. 2005, GCN Circ. 3054

Granot, J., Königl, A., \& Piran, T. 2006, MNRAS, 370, 1946

Gronwall, C., et al. 2005, GCN Circ. 3057

Groot, P. J., et al. 1998a, ApJ, 502, L123 1998b, ApJ, 493, L27

Holland, S. T. 2006, GCN Circ. 5158

Holland, S. T., et al. 2005, GCN Circ. 3150

Hullinger, D., et al. 2005, GCN Circ. 3364

Hurkett, C., Barthelmy, S., Cummings, J., Gehrels, N., Krimm, H., Markwardt, C., \& Palmer, D. 2005a, GCN Circ. 3360

Hurkett, C., et al. 2005b, GCN Circ. 3379

Ivanushkina, M., et al. 2005, GCN Circ. 3046

Jakobsson, P., et al. 2004, ApJ, 617, L21 (J04)

2006, A\&A, 447, 897

Kahharov, B., Ibrahimov, M., Sharapov, D., Pozanenko, A., Rumyantsev, \& V., \& Beskin, G. 2005, GCN Circ. 3274

Karimov, R., Ibrahimov, M., Kahharov, B., Asfandiyarov, I., Sharapov, D., Pozanenko, A., Rumyantsev, V., \& Beskin, G. 2005, GCN Circ. 2958

Kehoe, R., et al. 2001, ApJ, 554, L159

Kelson, D., \& Berger, E. 2005, GCN Circ. 3101

Krimm, H., et al. 2005a, GCN Circ. 3111 2005b, GCN Circ. 3119

Kobayashi, S. 2000, ApJ, 545, 870

Kobayashi, S., Zhang, B., Mészáros, P., \& Burrows, D. N. 2005, preprint (astro-ph/0506157)

Kumar, P. 2000, ApJ, 538, L125

Kumar, P., \& Panaitescu, A. 2003, MNRAS, 346, 905

La Parola, V., et al. 2005, GCN Circ. 3218

Lazzati, D., Covino, S., \& Ghisellini, G. 2002, MNRAS, 330, 583

Li, W., Filippenko, A. V., Chornock, R., \& Jha, S. 2003, ApJ, 586, L9
Lipunov, V., et al. 2005, GCN Circ. 2986

Lloyd-Ronning, N. M., \& Zhang, B. 2004, ApJ, 613, 477

Lyutikov, M., \& Blandford, R. D. 2003, preprint (astro-ph/0312347)

MacMahon, E., et al, 2006, MNRAS, 366, 575

Madau, P. 1995, ApJ, 441, 18

Markwardt, C., et al. 2005, GCN Circ. 3143

Mason, K. O., et al. 2006, ApJ, 639, 311

McGowan, K., et al. 2005a, GCN Circ. 3317

2005b, GCN Circ. 3115

Mészáros, P., \& Rees, M. J. 1997a, ApJ, 476, 232 1997b, ApJ, 482, L29

Metzger, M., et al. 1997, Nature, 387, 879

Mulchaey, J., \& Berger, E. 2005, GCN Circ. 3114

Nakar, E., \& Piran, T. 2004, MNRAS, 353, 647

Nousek, J. A., et al. 2006, ApJ, 642, 389 (N06)

O'Brien, P. T., Willingale, R., Osborne, J. P., \& Goad, M. R. 2006, New J.

Phys., in press (astro-ph/0605230)

Page, K., et al. 2005, GCN Circ. 3027

Panaitescu, A., \& Kumar, P. 2002, ApJ, 571, 779

Panaitescu, A., Mészáros, P., Burrows, D., Nousek, J., Gehrels, N., O’Brien, P., \& Willingale, R. 2006a, MNRAS, 369, 2059

Panaitescu, A., Mészáros, P., Gehrels, N., Burrows, D., \& Nousek, J. 2006b, MNRAS, 366, 1357

Parsons, A., et al. 2005a, GCN Circ. 3094 2005b, GCN Circ. 3180

Piro, L., et al. 2002, ApJ, 577, 680

Rees, M. J., \& Mészáros, P. 1998, ApJ, 496, L1

Retter, A., Parsons, A., Gehrels, N., Gronwall, C., Markwardt, C. B., \& Palmer, D. 2005 , GCN Circ. 3509

Rol, E., Wijers, R. A. M. J., Kouveliotou, C., Kaper, L., \& Kaneko, Y. 2005a, ApJ, 624, 868

Rol, E., et al. 2005b, GCN Circ. 3186

Romano, P., Perri, M., Beardmore, A., Mangano, V., Burrows, D. N., Hill, J. E., Zhang, B., \& Gehrels, N. 2005, GCN Circ. 3036

Roming, P. W. A., \& Mason, K. O. 2006, in AIP Conf. Proc. 836, 16th Maryland Astrophys. Conf. on Gamma-Ray Bursts in the Swift Era, ed. S. S.

Holt, N. Gehrels, \& J. A. Nousek (Melville: AIP), 224 (RM06)

Roming, P. W. A., et al. 2005a, Space Sci. Rev., 120, 95 2005b, GCN Circ. 3026 2005 c, GCN Circ. 3249

Rosen, S., Hurkett, C., Landsman, W., Roming, P., Poole, T., Gehrels, N., Mason, K., \& Nousek, J. 2005a, GCN Circ. 3367

Rosen, S., et al. 2005b, GCN Circ. 3095

Rykoff, E., Schaefer, B., \& Quimby, R. 2005a, GCN Circ. 3116

Rykoff, E. S., Yost, S. A., \& Swan, H. 2005b, GCN Circ. 3465

Sakamoto, T., et al. 2005, GCN Circ. 3264

Schady, P., Sakamoto, T., McGowan, K., Boyd, P., Roming, P., Nousek, J., \& Gehrels, N. 2005a, GCN Circ. 3276

Schady, P., et al. 2005b, GCN Circ. 3039 2006a, MNRAS, submitted 2006b, ApJ, 643, 276

Sharapov, D., Ibrahimov, M., Karimov, R., Kahharov, B., Pozanenko, A., Rumyantsev, V., \& Beskin, G. 2005, GCN Circ. 3124

Spruit, H. C., Daigne, F., \& Drenkhahn, G. 2001, A\&A, 369, 694

Still, M., et al. 2005, ApJ, 635, 1187

Suzuki, M., et al. 2005, GCN Circ. 3316

Tagliaferri, G., et al. 2005, Nature, 436, 985

Tanvir, N., et al. 2005a, GCN Circ. 3031 2005b, GCN Circ. 3375

Taylor, G. B., Bloom, J. S., Frail, D. A., Kulkarni, S. R., Djorgovski, S. G., \& Jacoby, B. A. 2000, ApJ, 537, L17

Taylor, G. B., et al. 1998, ApJL, 502, L115

Thompson, C. 1994, MNRAS, 270, 480

Uhm, Z, \& Beloborodov, A. M. 2006, in AIP Conf. Proc. 836, 16th Maryland Astrophys. Conf. on Gamma-Ray Bursts in the Swift Era, ed. S. S. Holt, N. Gehrels, \& J. A. Nousek (Melville: AIP), 189

Usov, V. V. 1992, Nature, 357, 472

Van Paradijs, J., et al. 1997, Nature, 386, 686

Vestrand, W. T., et al. 2005, Nature, 435, 178

Yost, S. A., et al. 2006, in AIP Conf. Proc. 836, 16th Maryland Astrophys. Conf. on Gamma-Ray Bursts in the Swift Era, ed. S. S. Holt, N. Gehrels, \& J. A. Nousek (Melville: AIP), 349

Zhang, B., Fan, Y. Z., Dyks, J., Kobayashi, S., Mészáros, P., Burrows, D. N., Nousek, J. A., \& Gehrels, N. 2006a, ApJ, 642, 354 (Z06a)

Zhang, B., \& Kobayashi, S. 2005, ApJ, 628, 315

Zhang, B., Kobayashi, S., \& Mészáros, P. 2003, ApJ, 595, 950

Zhang, B., \& Mészáros, P. 2002, ApJ, 571, 876

Zhang, B., et al. 2006b, ApJ, in press (astro-ph/0610177) (Z06b) 